\title{
Interleukin (IL)-1 family cytokine in primary immune thrombocytopenia: a comparison with systemic lupus erythematosus-associated thrombocytopenia
}

\section{Yanxia Zhan}

Zhongshan Hospital

\section{Boting Wu}

Zhongshan hospital

Chanjuan liu

Zhongshan Hospital

\section{Luya Cheng}

Zhongshan Hospital

Lili Ji

Zhongshan Hospital

\section{Feng Li}

Zhongshan Hospital Fudan University

\section{Yang Ke}

Zhongshan Hospital Fudan University

\section{Pu Chen}

Zhongshan Hospital Fudan University

\section{Fanli Hua}

Zhongshan Hospital Fudan University

\section{Ling Yuan}

Zhongshan Hospital Fudan University

\section{Zhihui Min}

Zhongshan Hospital Fudan University

\section{Lihua Sun}

Zhongshan Hospital

\section{Hao Chen}

Zhongshan Hospital Fudan University

\section{Yunfeng Cheng ( $\nabla$ yfcheng@fudan.edu.cn )}

Zhongshan Hospital, Fudan University https://orcid.org/0000-0002-9581-1134 
Keywords: Primary immune thrombocy topenia, Systemic lupus erythematosus, IL 1 cytokine family, Biomarker

Posted Date: March 18th, 2020

DOI: https://doi.org/10.21203/rs.3.rs-17605/v1

License: (c) (1) This work is licensed under a Creative Commons Attribution 4.0 International License. Read Full License 
Interleukin (IL)-1 family cytokine in primary immune thrombocytopenia: a comparison with systemic lupus

\title{
erythematosus-associated thrombocytopenia
}

Yanxia Zhan, BS ${ }^{1}$, Boting Wu, MD, $\mathrm{PhD}^{2}$, Chanjuan Liu, $\mathrm{MD}^{1}$, Luya Cheng, $\mathrm{MD}^{1}$,

Lili Ji, MD, $\mathrm{PhD}^{1}$, Feng Li, MD, $\mathrm{PhD}^{1,3}$, Yang Ke, $\mathrm{MD}^{1}$, Pu Chen, $\mathrm{MD}^{4}$, Fanli Hua, $\mathrm{MD}, \mathrm{PhD}^{3}$, Ling Yuan, MD ${ }^{1}$, Zhihui Min, $\mathrm{MD}^{5}$, Lihua Sun, $\mathrm{MD}^{3}$, Hao Chen, $\mathrm{MD}, \mathrm{PhD}^{6}$, Yunfeng Cheng, $\mathrm{MD}, \mathrm{PhD}^{1,3,5,7^{*}}$

1. Department of Hematology, 2. Department of Transfusion Medicine, 4. Department of Clinical Laboratory, 5. Institute of Clinical Science, Zhongshan Hospital Fudan University, Shanghai 200032, China

3. Department of Hematology, Zhongshan Hospital Qingpu Branch, Fudan University, Shanghai, 201700, China

6. Department of Thoracic Surgery, Zhongshan Hospital Xuhui Branch, Fudan University, Shanghai, 200031, China

7. Center for Tumor Diagnosis \& Therapy, Jinshan Hospital, Fudan University, Shanghai, 201508, China

Short Title: IL-1 family cytokine in ITP and SLE

Keywords: Primary immune thrombocytopenia; Systemic lupus erythematosus; IL-1 cytokine family; Biomarker

\author{
*Corresponding author \\ Yunfeng Cheng, MD, PhD, \\ Professor of Medicine \\ Institute of Clinical Science, Department of Hematology, \\ Zhongshan Hospital, Fudan University,
}


180 Fenglin Rd, Shanghai 200032, China

Telephone: +86-21-60267312

Email: yfcheng@fudan.edu.cn

\section{Authors' emails}

Yanxia Zhan: zhan.yanxia@ zs-hospital.sh.cn

Boting Wu: wu.boting@zs-hospital.sh.cn

Chanjuan liu: 15111210015@fudan.edu.cn

Luya Cheng: cheng.luya@ zs-hospital.sh.cn

Lili Ji: ji.lili@ zs-hospital.sh.cn

Feng Li: li.feng@zs-hospital.sh.cn

Yang Ke: ke.yang@zs-hospital.sh.cn

Pu Chen: chen.pu@zs-hospital.sh.cn

Fanli Hua: $\underline{\text { hua_fanli@fudan.edu.cn }}$

Ling Yuan: yuan.ling@zs-hospital.sh.cn

Zhihui Min: min.zhihui@zs-hospital.sh.cn

Lihua Sun: qpsunlh023@126.com

Hao Chen: h.chen@fudan.edu.cn 


\section{Abstract}

Background: Primary immune thrombocytopenia (ITP) is an autoimmune-mediated disorder characterized by decreased platelet count. Systemic lupus erythematosus (SLE) is also an autoimmune disease which thrombocytopenia is a common hematologic manifestation. Interleukin (IL)-1 family cytokines are major proinflammatory and immunoregulatory mediators. This study aimed to investigate the role of IL-1 cytokines in patients with ITP and SLE and the potential pathophysiologic mechanism to differentiate SLE-associated thrombocytopenia (SLE-TP) from ITP.

Methods: Multiplex cytokine assay and real-time polymerase chain reaction (RT-PCR) were used to measure the IL-1 cytokines in 17 newly diagnosed ITP patients, 17 SLE-TP patients, 19 SLE patients without thrombocytopenia (SLE-NTP) and 10 healthy controls.

Results: The serum levels of IL-1 $\beta$, IL-18, IL-36 $\alpha$, IL-36 $\beta$, IL-36 $\gamma$ and IL-33 were decreased significantly in ITP patients as compared with SLE-TP, SLE-NTP patients and healthy controls $(p<0.05)$. There was no significantly difference in the serum level of IL-37 between ITP and SLE-TP patients, however, there is a positive correlation between platelet count with IL-37 level in ITP patients. Our data suggested that serum IL-1 $\beta$, IL-18, IL-36 $\alpha$, IL-36 $\beta$, IL-36 $\gamma$, IL-33 and IL-37 were involved in the pathogenesis of ITP.

Conclusions: Serum IL-1 $\beta$, IL-18, IL-36 $\alpha$, IL-36 $\beta$, IL-36 $\gamma$ and IL-33 could be considered biomarkers to differentiate SLE-TP from ITP patients. 


\section{Background}

Primary immune thrombocytopenia (ITP) is an autoimmune-mediated acquired bleeding disorder, defined as a platelet count less than $100 \times 10^{9} / \mathrm{L}$ without other causes of isolated thrombocytopenia $[1,2]$. The etiology of ITP is complex and heterogeneous. At present, the diagnosis of ITP is still exclusive diagnosis, and there is lack of molecular markers specific to diagnosis. Multiple mechanisms have been described in ITP such as autoantibodies, $\mathrm{T}$ cell dysregulation and impaired Megakaryocyte[1, 2], highlighting the heterogeneous nature of ITP. Because of the heterogeneous nature of ITP, clinical presentation and the response to regular treatment are different among patients. Several causes or diseases such as systemic lupus erythematosus (SLE) are associated with thrombocytopenia, defined as secondary ITP.

SLE is a complex autoimmune disease and is usually associated with hematological abnormality[3], also with thrombocytopenia. Prevalence of thrombocytopenia in SLE patients has been reported to be 7\%-30\% [4]. Conversely, prevalence of SLE in all ITP cases in adult is approximately 5\%, which is the most common cause of secondary ITP[5]. Sometimes in the early stages of SLE, when there are only symptoms of thrombocytopenia, it is difficult to differentiate between the ITP with SLE[5]. SLE-associated thrombocytopenia (SLE-TP) is defined as a platelet count less than $100 \times 10^{9} / \mathrm{L}$ in the absence of any other identifiable cause[6]. The pathogenesis of thrombocytopenia in SLE is heterogeneous and multifactorial. However, it is widely accepted that increased platelet clearance mediated by 
autoantibody against platelet contributes to the pathogenesis, which is analogous to the mechanism of ITP. Different from primary ITP, clinical treatment for thrombocytopenia secondary to an identifiable cause is often targeted toward the ongoing disorder. However, the biomarker to differentiate SLE-TP from ITP is still not very clear [7-9].

The family of interleukin 1 (IL-1) cytokines consists of 11 members, including 7 pro-inflammatory agonist, IL-1 $\alpha$ (IL-1F1), IL-1 $\beta$ (IL-1F2), IL-18 (IL-1F4), IL-33

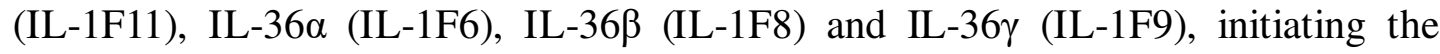
proinflammatory signal and 4 antagonists or anti-inflammatory activity, IL-1 receptor antagonist (IL-1Ra, IL-1F3), IL-36Ra (IL-1F5), IL-37 (IL-1F7) and IL-38 (IL-1F10), inhibiting inflammation or trigger anti-inflammatory reactions[10]. IL-1 cytokine family is a family of protein molecules that play a crucial role as a major proinflammatory and immunoregulatory mediator in a wide range of autoinflammatory, infectious, tumor and autoimmune disease which act through receptors of the Toll-like/IL-1 receptor superfamily[11-15]. The productions of inflammatory cytokines such as IL-1, IL-36 and IL-18 activate target cells through the receptors superfamily then amplify the immune response. However, the antagonist such as IL-1Ra is the receptor antagonist of IL-1, including IL-1 $\alpha$ and IL-1 $\beta$, which act as an inhibitor of IL-1 dependent inflammation.

Recently, IL-1 family cytokines have gained extra attention. It has been reported that blocking IL-1, particularly IL-1 $\beta$, is now the standard of therapy for autoinflammatory diseases[16, 17]. Moreover, IL-1 $\beta$, driving tumor-promoting 
inflammation in cancer, can be targeted in patients by using an IL-1 receptor antagonist which can be considered a checkpoint inhibitor[18, 19]. Several studies have described that the abnormal changes of IL-18 and IL-18-binding protein (IL-18BP) were involved in the pathogenesis of SLE and ITP[20-23]. Furthermore, recent studies demonstrate that IL-1 may also take part in inflammatory pathologies and auto-immune diseases through participate in the development of T-helper 17 (Th17) cells[24, 25]. Increased numbers of Th17 cells have been reported in the patients with SLE and ITP. Because of the role of IL-1 cytokines in inflammation and autoimmune diseases, we hypothesize that IL-1 family cytokines could be involved in the pathogenesis of ITP and/or SLE. In the present study, we aimed to evaluate the expression of IL-1 family cytokines in ITP patients, SLE-TP patients and SLE without thrombocytopenia (SLE-NTP) patients, which might help to differentiate SLE-TP patients from ITP patients.

\section{Materials and methods}

\section{Patients and controls}

Seventeen patients with newly diagnosed primary ITP (10 females and 7 males, median age 52 years, range 23-71 years), seventeen patients with SLE-TP (15 females and 2 males, median age 36 years, range 21-61 years), nineteen patients with SLE-NTP (15 females and 4 males, median age 36 years, range 17-66 years) were enrolled in the study. These patients were recruited from Zhongshan Hospital of Fudan University (Shanghai, China) between July 2014 and May 2018, fulfilling the diagnosis criteria of ITP updated by an international working group [1] and the 
diagnosis of SLE according to the European League Against Rheumatism Executive Committee and the American College of Rheumatology criteria [5], respectively. Also 10 age- and sex-matched healthy controls were enrolled in the study (6 females and 4 males, median age 40.5 years, range $24-68$ years). The study was approved by local Medical Ethics Committees of Zhongshan Hospital of Fudan University. Written informed consent was obtained from each patient in accordance with the declaration of Helsinki before being included in the study.

\section{Multiplex cytokine assay}

Blood samples were obtained from ITP patients, SLE patients and healthy controls. The blood samples were allowed to clot at room temperature for $1 \mathrm{~h}$, and serum was removed by centrifugation $\left(1500 \mathrm{~g}, 10\right.$ minutes). The serum was stored at $-80^{\circ} \mathrm{C}$ until analyzed. Serum levels of IL-1 cytokine family, including IL-1 $\alpha$, IL-1 $\beta$, IL-1Ra, IL-18, IL-36Ra, IL-36 $\alpha$, IL-37, IL-36 $\beta$, IL-36 $\gamma$, IL-38 and IL-33 were measured by Quantibody Human IL-1 Family Cytokine Array 1 (RayBiotech, USA), according to the manufacturer's protocol. The signals (green fluorescence, Cy3 channel, 555-nm excitation, and 565-nm emission) were captured using a LuxScan-10K/A (Capitalbio,

China). Quantitative data analysis was performed using RayBio Q Analyzer Software (RayBiotech, USA).

\section{Isolation of peripheral blood mononuclear cells (PBMCs)}

Ethylenediaminetetraacetic acid (EDTA)-anticoagulated venous blood samples were collected and diluted 1:2 with Hanks balanced salt solution (HBSS) before Ficoll-Hypaque gradient centrifugation (2500rpm at room temperature for $20 \mathrm{~min}$ ). 
Washed and resuspended in fetal bovine serum containing $10 \%$ dimethyl sulfoxide (DMSO) and stored in $-80^{\circ} \mathrm{C}$ until RNA extraction.

\section{Real-Time Polymerase Chain Reaction (RT-PCR)}

Total RNA was isolated from PBMCs of each sample by using Trizol reagent (Invitrogen, USA) and converted into cDNA using a PrimeScript RT Reagent Kit (Takara, Japan) following the manufacturer's instructions. The mRNA expressions of IL-1 cytokines were quantified using the TB Green Premix Ex Taq (Takara, Japan) on a 7500 Real Time PCR System (Applied Biosystems, USA). Amplification was performed for 40 cycles of $5 \mathrm{~s}$ at $95^{\circ} \mathrm{C}$ and $34 \mathrm{~s}$ at $60^{\circ} \mathrm{C}$ after initial denaturation $\left(95^{\circ} \mathrm{C}, \quad 30 \mathrm{~s}\right)$. The primers used were as follows: IL-1 $\alpha$, 5'-TGAAGCCGAGCCTCAAGATGAAG-3', and 5'-GGATGCCTGGTCACACTCAGAG-3'; IL-1 $\beta$, 5'-ACAGTGGCAATGAGGATGACTTGT-3' and

5'-TGtAGTGGTGGTCGGAGATTCGTA-3'; IL-1Ra,

5'-TGTGCCTGTCCTGTGTCAAGTC-3'

5'-GCCACTGTCTGAGCGGATGAAG-3'; IL-18,

5'-GCCTGGACAGTCAGCAAGGAATT-3' and

5'-GCGAGAGGAAGCGATCTGGAAG-3'; IL-36Ra,

5'-GGAAGCCAGTGCCTGTCATGTG-3', and

5'-TCCCGCCGGTAGAAGGTGAAG-3'; IL-36 5'-CGAGGAAGGACCGTATGT-3' and 5'-TGGCTGTGGTAGAAGAGAA-3'; IL-37, 5'-GCTCTGAGGACTGGGAAAAAGAT-3' 
5'-TCACCTTTGGACTTGTGTGAAC-3';

IL-36 $\beta$,

5'-TTCCTAGCCTCCTCACCACCATC-3'

and

5'-CCACTCAGGACCCACACCATCT-3';

IL-36 $\gamma$

5'-TGGTTCATTGCCTCCTCCAAGAGA-3'

and

5'-GCCACATCCTGTTCTTCAGTTGCT-3';

IL-38,

5'-GCCACACGCTTCACCTTCTTCC-3'

and

5'-ACGCAGTTTCCTGTCTCCCTACC-3';

IL-33,

5'-GAAGCTCCGCTCTGGCCTTATG-3'

and

5'-CTGTTGACAGGCAGCGAGTACC-3';

GAPDH,

5'-TCGGAGTCAACGGATTtGGT-3' and 5'-TTCCCGTTCTCAGCCTTGAC-3'.

Data were normalized to the level of GAPDH mRNA. The formula $2^{-\triangle \triangle} \mathrm{Ct}$ was used to analyze fold change of mRNA expression.

\section{Statistical analysis}

Analysis was performed with the SPSS 19.0 and GraphPad Prism 5.0 for Windows.

For statistical analysis, the levels of cytokine below the detection limits were arbitrarily assigned the values corresponding to the minimum limits. Data were expressed as median (interquartile range). Differences in percentages were evaluated using chi-square analysis or the Fisher's exact tests. Differences among the groups in measured values were analyzed using the one-way ANOVA with the least significant difference test for post-hoc multiple comparisons, Kruskal-Wallis test and Mann-Whitney $\mathrm{U}$ test as appropriate. $P$-values less than 0.05 were considered statistically significant. 


\section{Results}

\section{Patient characteristics}

Seventeen newly diagnosed primary ITP patients with a median platelet count $9 \times 10^{9} / \mathrm{L}$, range $1-22 \times 10^{9} / \mathrm{L}$. Seventeen SLE-TP patients with a median platelet count $66 \times 10^{9} / \mathrm{L}$, range $29-95 \times 10^{9} / \mathrm{L}$. Nineteen SLE-NTP patients with a median platelet count $183 \times 10^{9} / \mathrm{L}$, range $104-327 \times 10^{9} / \mathrm{L}$. Ten age- and sex-matched healthy controls with a median platelet count $247 \times 10^{9} / \mathrm{L}$, range $185-309 \times 10^{9} / \mathrm{L}$. Characteristics of the cases enrolled in the study are shown in Table 1. There were no significant differences in terms of distributions on age and sex among the ITP patients, SLE-TP patients, SLE-NTP patients and the controls $(p>0.05)$.

Serum IL-1 family cytokines in primary ITP patients, SLE-TP patients and SLE-NTP patients correlate with disease activity.

In order to understand the serum IL-1 cytokines profile in ITP patients, SLE-TP patients and SLE-NTP patients, eleven cytokines including IL-1 $\alpha$, IL-1 $\beta$, IL-1Ra, IL-18, IL-36Ra, IL-36 $\alpha$, IL-37, IL-36 $\beta$, IL-36 $\gamma$, IL-38 and IL-33 were analyzed (Table 2). The majority of IL-1 $\alpha$ levels were below the detection limits of the assay (data not shown). Six pro-inflammatory cytokines were shown in Figure 1. As shown

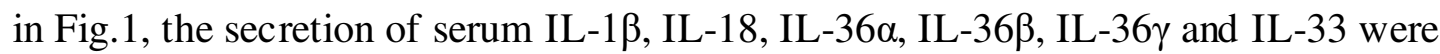
significantly decreased in primary ITP patients as compared with SLE-TP, SLE-NTP patients and healthy controls (all $p<0.05$ ). However, there were no significantly differences between the SLE-NTP patients and SLE-TP patients/health controls $(p>0.05)$. Except IL-33, there were no significantly differences between the SLE-TP 
patients and health controls.

Four anti-inflammatory cytokines were shown in Figure 2. As demonstrated in Fig.2, IL-1Ra and IL-36Ra were increased in primary ITP and SLE-TP patients than healthy controls without significant differences. There was significantly difference in the secretion of IL-1Ra between the primary ITP and SLE-TP patients. As compared with the controls, IL-37 and IL-38 were decreased significantly in primary ITP patients and SLE-TP patients. The level of IL-37 was increased significantly in SLE-NTP patients, compared with SLE-TP patients.

The secretion of serum IL-37 and IL-36 $\alpha$ correlated positively to platelet count in primary ITP and SLE-NTP patients, respectively

IL-37 positively correlated with platelet count was found in primary ITP $(r=0.617$, $p=0.008$, Figure 3A). No significant correlation was found between IL-1 cytokines and platelet count in SLE-TP patients, but a significant positive correlation was found between IL-36 $\alpha$ and platelet count in SLE-NTP patients $(r=0.673, p=0.002$, Figure 3B).

The change of IL-1 mRNA expression between primary ITP patients and SLE-TP patients are not similar to those observed at the protein level To better understand the expression of IL-1 cytokines in primary ITP patients, SLE-TP patients and SLE-NTP patients, we next analyzed the mRNA expression of IL-1 cytokines. The expression of IL-1 $\beta$ mRNA was decreased significantly in SLE-TP patients as compared with SLE-NTP and controls. However, the expression of IL-36 3 and IL-38 mRNA were increased significantly in SLE-TP patients as 
compared with SLE-NTP and controls. In addition, there were no significantly differences between primary ITP patients and SLE-TP patients (Figure 4). The expression patterns of IL-1 mRNA expression were not similar to those that observed at the protein levels in ITP patients and SLE-TP patients.

\section{Discussion}

Immune thrombocytopenia is an autoimmune disease with abnormal $\mathrm{T}$ cell immunity[26]. Abnormal cytokines play an important role in its pathogenesis. The role of IL-1 family cytokines in the development of autoinflammatory and autoimmune diseases has gained extra attention because of their potential therapeutic targets. However, there was a few of IL-1 cytokines related to thrombocytopenia have been described previously; the majority of these significant cytokines have not been identified in ITP and SLE. Increasing evidences suggest that imbalance of immune regulation mechanisms, such as Th1/Th2 imbalance, Treg/Th17 imbalance, pro-inflammatory cytokines and anti-inflammatory cytokines imbalance, contribute to the maintenance of immune homeostasis[27]. Both ITP and SLE have been reported with increased Th17 cells. Moreover, IL-1 may participate in the development of Th17 cells. It is intriguing that we observed a down-regulation of IL-1 $\beta$, IL-18, IL-36 $\alpha$, IL-36 $\beta$, IL-36 $\gamma$, IL-33, IL-37 and IL-38 in ITP patients as compared with healthy controls. The levels of IL-18 and IL-33 in serum were significantly down-regulated in ITP patients, which is in accordance with previous reports[28]. IL-18 is best known for its role in Th1 immune responses, whereas IL-33 has been strongly associated with Th2 responses. These findings support the 
hypothesis that IL-1 cytokines participate in the pathogenesis of ITP.

The derangement of immune regulation also contributes to SLE. SLE-TP is cause by the autoantibody against platelet, which is analogous to ITP. The mechanism to differentiate SLE-TP from ITP remains unknown. In this study, we first detected the IL-1 cytokines among ITP patients, SLE-TP patients and SLE-NTP patients. The assessment of IL-1 cytokines in ITP patients in comparison with SLE-TP and SLE-NTP showed that the levels of IL-1 $\beta$, IL-18, IL-36 $\alpha$, IL-36 $\beta$, IL-36 $\gamma$ and IL-33 were significantly lower in ITP patients than SLE-TP and SLE-NTP patients. However, there were no significant differences in the levels of IL-1 $\beta$, IL-18, IL-36 $\alpha$, IL-36 $\beta$ and IL-36 $\gamma$ between SLE-TP/SLE-NTP patients and healthy controls, also between SLE-TP patients and SLE-NTP patients. These data reveal a number of differences in the immunopathogenesis between ITP and SLE-TP/SLE-NTP patients. These findings indicate that IL-1 $\beta$, IL-18, IL-36 $\alpha$, IL-36ß, IL-36 $\gamma$ and IL-33 involved in the pathogenesis of ITP but not SLE, including SLE-TP and SLE-NTP. Although there was no significantly difference in the level of serum IL-37 between ITP and SLE-TP patients, a significantly positive correlation was found between IL-37 and platelet count in ITP patients. Serum IL-1 $\beta$, IL-18,

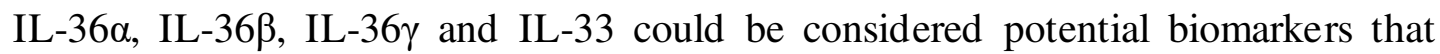
discriminated between ITP and SLE-TP patients.

To better assess the expression of IL-1 cytokines, we also analyzed the mRNA expression of IL-1 cytokines among patients. What is interesting, there were no significantly differences between ITP and SLE-TP patients, which were not similar 
to those that observed at the protein levels, leading us to conclude that post-transcriptional regulation such as microRNAs may take part in the pathogenesis of ITP and SLE-TP patients.

In conclusion, the present study indicates that parts of the IL-1 cytokines, such

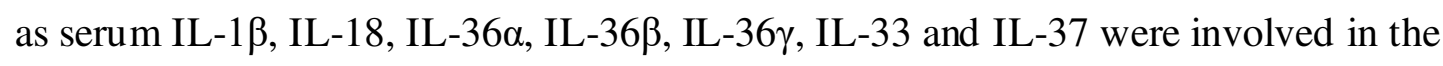
pathogenesis of ITP. The protein levels of IL-1 $\beta$, IL-18, IL-36 $\alpha$, IL-36 $\beta$, IL-36 $\gamma$ and IL-33 rather than mRNA levels could be considered the biomarkers to differentiate SLE-TP from ITP patients. Considering the role of IL-1 cytokines in autoimmune and autoinflammation function, further investigations of IL-1 cytokines are warranted.

\section{Competing interests}

The authors declare that there is no conflict of interests regarding the publication of this paper.

\section{Consent for publication}

Not applicable.

\section{Availability of data and materials}

Not applicable.

\section{Ethics approval and consent to participate}

The study was in accordance with the ethical standards formulated in the Helsinki Declaration and was approved by the respective local Medical Ethics Committees of Zhongshan Hospital of Fudan University. Written informed consent was obtained from each patient before being included in the study.

\section{Funding}


This work was supported by grants from National Natural Science Foundation of China (81870098, 81600090, 81500090, and 81470282), Shanghai Health Bureau Funding (201840351, and 20144Y0194), and the Science and Technology Commission of Shanghai Municipality (18ZR1407200). All authors obtain permission to acknowledge from all those mentioned in the Acknowledgements.

\section{Acknowledgements}

This study was conducted at the Institute of Clinical Science of Zhongshan Hospital, Fudan University. We sincerely thank all the staff and participants for their important contributions.

\section{Contributorship statement}

Yanxia Zhan, Boting Wu, and Yunfeng Cheng conceived the study; Chanjuan Liu, Luya Cheng, Feng Li and Yunfeng Cheng performed the literature review, drafted and revised the manuscript; Hao Chen and Yunfeng Cheng contributed to the critical revision of the manuscript; Pu Chen, Fanli Hua, Yanxia Zhan, Zhihui Min, Yang Ke, Ling Yuan, Lili Ji and Lihua Sun performed the experiments, analyzed data. All authors read and approved the final manuscript. 


\section{References:}

1. Provan D, Arnold DM, Bussel JB, Chong BH, Cooper N, Gernsheimer T, Ghanima W, Godeau B, Gonzalez-Lopez TJ, Grainger J, et al: Updated international consensus report on the investigation and management of primary immune thrombocytopenia. Blood Adv 2019, 3:3780-3817.

2. Cooper N, Ghanima W: Immune Thrombocytopenia. N Engl J Med 2019, 381:945-955.

3. Wu B, Wang W, Zhan Y, Li F, Zou S, Sun L, Cheng Y: CXCL13, CCL4, and sTNFR as circulating inflammatory cytokine markers in primary and SLE-related autoimmune hemolytic anemia. J Transl Med 2015,13:112.

4. Velo-Garcia A, Castro SG, Isenberg DA: The diagnosis and management of the haematologic manifestations of lupus. J Autoimmun 2016, 74:139-160.

5. Cines DB, Bussel JB, Liebman HA, Luning Prak ET: The ITP syndrome: pathogenic and clinical diversity. Blood 2009, 113:6511-6521.

6. Aringer M, Costenbader K, Daikh D, Brinks R, Mosca M, Ramsey-Goldman R, Smolen JS, Wofsy D, Boumpas DT, Kamen DL, et al: 2019 European League Against Rheumatism/American College of Rheumatology Classification Criteria for Systemic Lupus Erythematosus. Arthritis Rheumatol 2019, 71:1400-1412.

7. Altintas A, Ozel A, Okur N, Okur N, Cil T, Pasa S, Ayyildiz O: Prevalence and clinical significance of elevated antinuclear antibody test in children and adult patients with idiopathic thrombocytopenic purpura. J Thromb Thrombolysis 2007, 24:163-168.

8. Kuwana M, Kaburaki J, Okazaki Y, Miyazaki H, Ikeda Y: Two types of autoantibody-mediated thrombocytopenia in patients with systemic lupus ery thematosus. Rheumatology (Oxford) 2006, 45:851-854.

9. Lazarus AH, Ellis J, Semple JW, Mody M, Crow AR, Freedman J: Comparison of platelet immunity in patients with SLE and with ITP. Transfus Sci 2000, 22:19-27.

10. Dinarello CA: Interleukin-1 in the pathogenesis and treatment of inflammatory diseases. Blood 2011, 117:3720-3732.

11. Fields JK, Gunther S, Sundberg EJ: Structural Basis of IL-1 Family Cytokine Signaling. Front Immunol 2019, 10:1412.

12. Baker KJ, Houston A, Brint E: IL-1 Family Members in Cancer; Two Sides to Every Story. Front Immunol 2019, 10:1197.

13. Dinarello CA, Simon A, van der Meer JW: Treating inflammation by blocking interleukin-1 in a broad spectrum of diseases. Nat Rev Drug Discov 2012, 11:633-652.

14. Gabay C, Lamacchia C, Palmer G: IL-1 pathways in inflammation and human diseases. Nat Rev Rheumatol 2010, 6:232-241.

15. Dinarello CA: Immunological and inflammatory functions of the interleukin-1 family. Annu Rev Immunol 2009, 27:519-550.

16. Simon A, van der Meer JW: Pathogenesis of familial periodic fever syndromes or hereditary autoinflammatory syndromes. Am J Physiol Regul Integr Comp Physiol 2007, 292:R86-98.

17. Masters SL, Simon A, Aksentijevich I, Kastner DL: Horror autoinflammaticus: the 
molecular pathophysiology of autoinflammatory disease (*). Annu Rev Immunol 2009, 27:621-668.

18. Ridker PM, MacFadyen JG, Thuren T, Everett BM, Libby P, Glynn RJ, Group CT: Effect of interleukin-1 beta inhibition with cana kinumab on incident lung cancer in patients with atherosclerosis: exploratory results from a randomised, double-blind, placebo-controlled trial. Lancet 2017,390:1833-1842.

19. Wu TC, Xu K, Martinek J, Young RR, Banchereau R, George J, Turner J, Kim KI, Zurawski S, Wang X, et al: IL1 Receptor Antagonist Controls Transcriptional Signature of Inflammation in Patients with Metastatic Breast Cancer. Cancer Res 2018, 78:5243-5258.

20. Calvani N, Tucci M, Richards HB, Tartaglia P, Silvestris F: Th1 cytokines in the pathogenesis of lupus nephritis: the role of IL-18. Autoimmun Rev 2005, 4:542-548.

21. Liang D, Ma W, Yao C, Liu H, Chen X: Imbalance of interleukin 18 and interleukin 18 binding protein in patients with lupus nephritis. Cell Mol Immunol 2006, 3:303-306.

22. Tucci M, Quatraro C, Lombardi L, Pellegrino C, Dammacco F, Silvestris F: Glomerular accumulation of plasmacytoid dendritic cells in active lupus nephritis: role of interleukin-18. Arthritis Rheum 2008, 58:251-262.

23. Shan NN, Zhu XJ, Peng J, Qin P, Zhuang XW, Wang HC, Hou M: Interleukin 18 and interleukin 18 binding protein in patients with idiopathic thrombocytopenic purpura. Br J Haematol 2009, 144:755-761.

24. Sutton C, Brereton C, Keogh B, Mills KH, Lavelle EC: A crucial role for interleukin (IL)-1 in the induction of IL-17-producing $T$ cells that mediate autoimmune encephalomyelitis. $J$ Exp Med 2006, 203:1685-1691.

25. Acosta-Rodriguez EV, Napolitani G, Lanzavecchia A, Sallusto F: Interleukins 1 beta and 6 but not transforming growth factor-beta are essential for the differentiation of interleukin 17-producing human T helper cells. Nat Immunol 2007, 8:942-949.

26. Ji $\mathrm{X}$, Zhang $\mathrm{L}$, Peng $\mathrm{J}$, Hou $\mathrm{M}$ : $\mathrm{T}$ cell immune abnormalities in immune thrombocytopenia. J Hematol Oncol 2014, 7:72.

27. Shao X, Wu B, Cheng L, Li F, Zhan Y, Liu C, Ji L, Min Z, Ke Y, Sun L, et al: Distinct alterations of CD68(+)CD163(+) M2 -like macrophages and myeloid-derived suppressor cells in newly diagnosed primary immune thrombocytopenia with or without $C R$ after high-dose dexamethasone treatment. J Transl Med 2018, 16:48.

28. Li PP, Zhang XM, Yuan D, Liu X, Li Y, Shan NN: Decreased expression of IL-33 in immune thrombocytopenia. Int Immunopharmacol 2015, 28:420-424. 
Table 1 Clinical characteristics of primary ITP, SLE-TP and SLE-NTP patients

\begin{tabular}{ccccc}
\hline Variables & $\begin{array}{c}\text { ITP } \\
(n=17)\end{array}$ & $\begin{array}{c}\text { SLE-TP } \\
(n=17)\end{array}$ & $\begin{array}{c}\text { SLE-NTP } \\
(n=19)\end{array}$ & $\begin{array}{c}\text { Controls } \\
(n=10)\end{array}$ \\
\hline $\begin{array}{c}\text { Median age } \\
\text { Sex }\end{array}$ & $52(23-71)$ & $36(21-61)$ & $36(17-66)$ & $40.5(24-68)$ \\
Female & 10 & 15 & 15 & 6 \\
Male & 7 & 2 & 4 & 4 \\
$\begin{array}{c}\text { Median platelet count } \\
\left(\times 10^{9} / \mathrm{L}\right)\end{array}$ & $9(1-22)$ & $66(29-95)$ & $183(104-327)$ & 247 (185-309) \\
\hline $\begin{array}{l}\text { Abbreviations: ITP, } \\
\text { erythematosus-associated thrombocytopenia; SLE-NTP, SLE without thrombocytopenia. }\end{array}$ & immune thrombocytopenia; & SLE-TP, systemic & lupus
\end{tabular}


Table 2 Serum IL-1 family cytokines levels of primary ITP, SLE-TP and SLE-NTP patients (pg/ml)

\begin{tabular}{|c|c|c|c|c|}
\hline & Primary ITP $(n=17)$ & $\operatorname{SLE}-\mathrm{TP}(n=17)$ & SLE-NTP $(n=19)$ & Health controls $(n=10)$ \\
\hline $\begin{array}{l}\text { IL-1F2 } \\
(\mathrm{IL}-1 \beta)\end{array}$ & $3.24(1.5-5.04){ }^{\text {※\# }}$ & $17.35(4.87-33.08)$ & $19.78(12.9-39.55)$ & $21.96(18.06-30.43)$ \\
\hline $\begin{array}{l}\text { IL-1F3 } \\
\text { (IL-1Ra) }\end{array}$ & $57.61(38.23-104.74){ }^{\#}$ & $21.3(10.03-75.62)$ & $24.12(12.58-48.48)$ & $31.11(19.84-55.1)$ \\
\hline $\begin{array}{l}\text { IL-1F4 } \\
\text { (IL-18) }\end{array}$ & $184.66(113.92-334.54)$ *\# & $\begin{array}{c}6935.64 \\
(281.53-9297.78)\end{array}$ & $8847.64(5663.6-10464.8)$ & $\begin{array}{c}7497.2 \\
(5967.43-8144.15)\end{array}$ \\
\hline $\begin{array}{l}\text { IL-1F5 } \\
\text { (IL-36Ra) }\end{array}$ & $1103.8(406.95-4497.75)$ & $\begin{array}{c}1150.49 \\
(131.35-3301.73)\end{array}$ & $4073.22(1884.32-4863.08) *$ & $246.05(77.23-997.78)$ \\
\hline $\begin{array}{l}\text { IL-1F6 } \\
\text { (IL-36 })\end{array}$ & $32.2(20-54.4){ }^{* \#}$ & 366.85 (91.88-939.57) & 499.7 (396.6-1055.81) & $790.7(366.63-1519.55)$ \\
\hline $\begin{array}{l}\text { IL-1F7 } \\
\text { (IL-37) }\end{array}$ & $23.49(20-28.35){ }^{\text {※\# }}$ & $30.29(22.06-38.67) *$ & $44.67(30.36-91.39){ }^{\#}$ & $83.96(27.1-238.27)$ \\
\hline $\begin{array}{l}\text { IL-1F8 } \\
\text { (IL-36 } 3)\end{array}$ & $1.04(0.5-1.28)$ ※\# & $10.97(2.56-35.15)$ & $17.26(4.62-30.25)$ & $19.79(16.58-38.93)$ \\
\hline 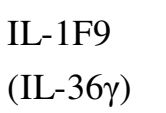 & $20(20-33.25){ }^{※ \#}$ & $1151.51(34.32-1422.48)$ & $1309.63(742.73-2276.75)$ & $\begin{array}{c}2074.9 \\
(1136.68-2649.83)\end{array}$ \\
\hline $\begin{array}{l}\text { IL-1F10 } \\
\text { (IL-38) }\end{array}$ & $36.84(29.89-47.16) *$ & $36.9(29.1-68.13) *$ & $47.21(35.73-92.29){ }^{*}$ & $569.97(366.32-691.12)$ \\
\hline $\begin{array}{l}\text { IL-1F11 } \\
\text { (IL-33) }\end{array}$ & $8.75(7-18.66){ }^{\text {※\# }}$ & $32.63(18.01-51.13) *$ & $45.59(27.7-68.05)$ & $46.99(38.16-115.9)$ \\
\hline
\end{tabular}

Abbreviations: ITP, immune thrombocytopenia; SLE-TP, systemic lupus
erythematosus-associated thrombocytopenia; SLE-NTP, SLE without thrombocytopenia. Data are presented as median (interquartile range). ${ }^{*} p<0.05$ compared to health control; ${ }^{*} p<0.05$ compared to SLE-TP. 
Figure 1 The serum levels of IL-1 $\beta$, IL-18, IL-36 $\alpha$, IL-36 $\beta$, IL-36 $\gamma$ and IL-33 in ITP patients, SLE-TP patients, SLE-NTP patients and healthy controls.
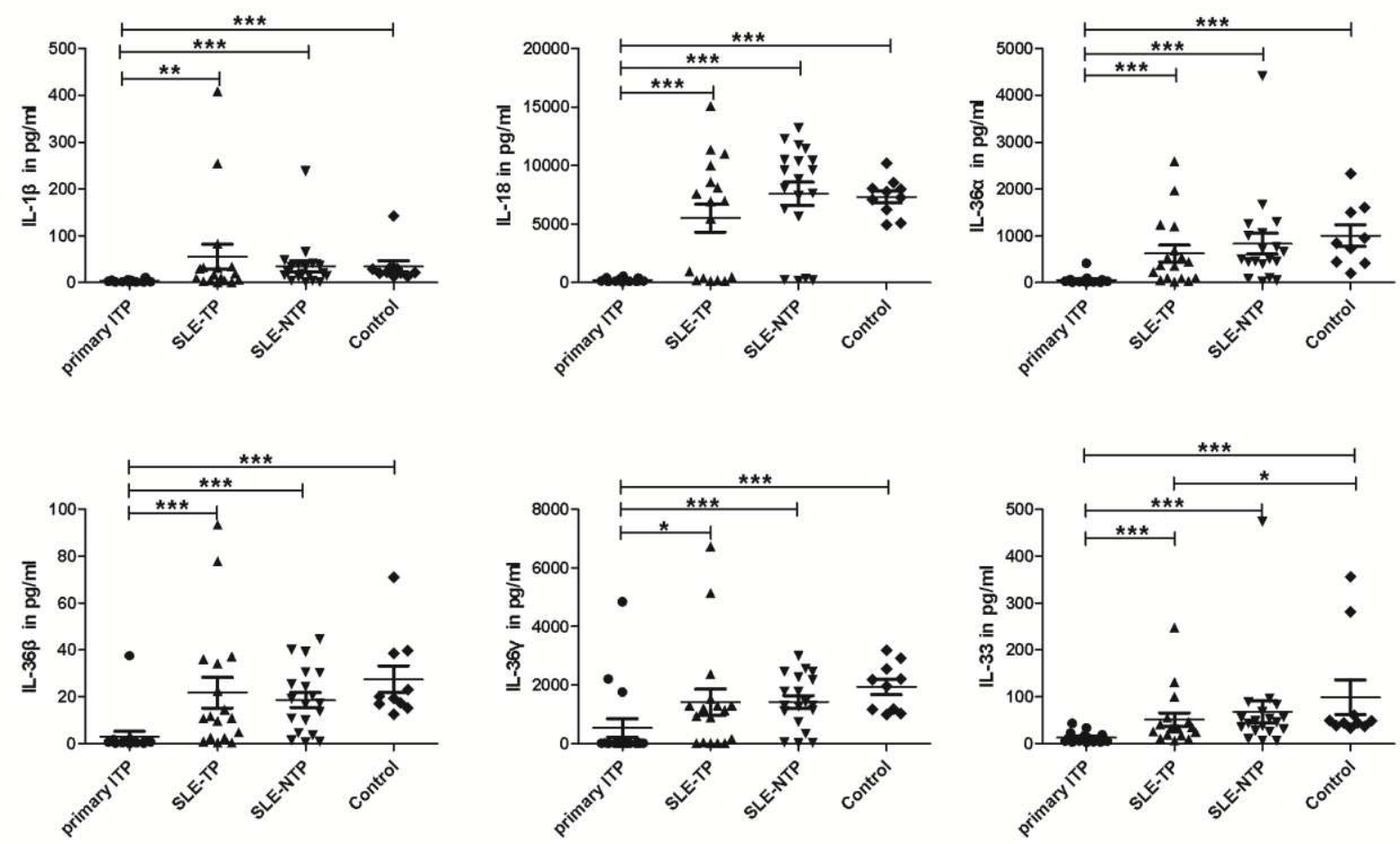
Figure 2 The levels of IL-1Ra, IL-36Ra, IL-37 and IL-38 in ITP patients, SLE-TP patients, SLE-NTP patients and healthy controls.
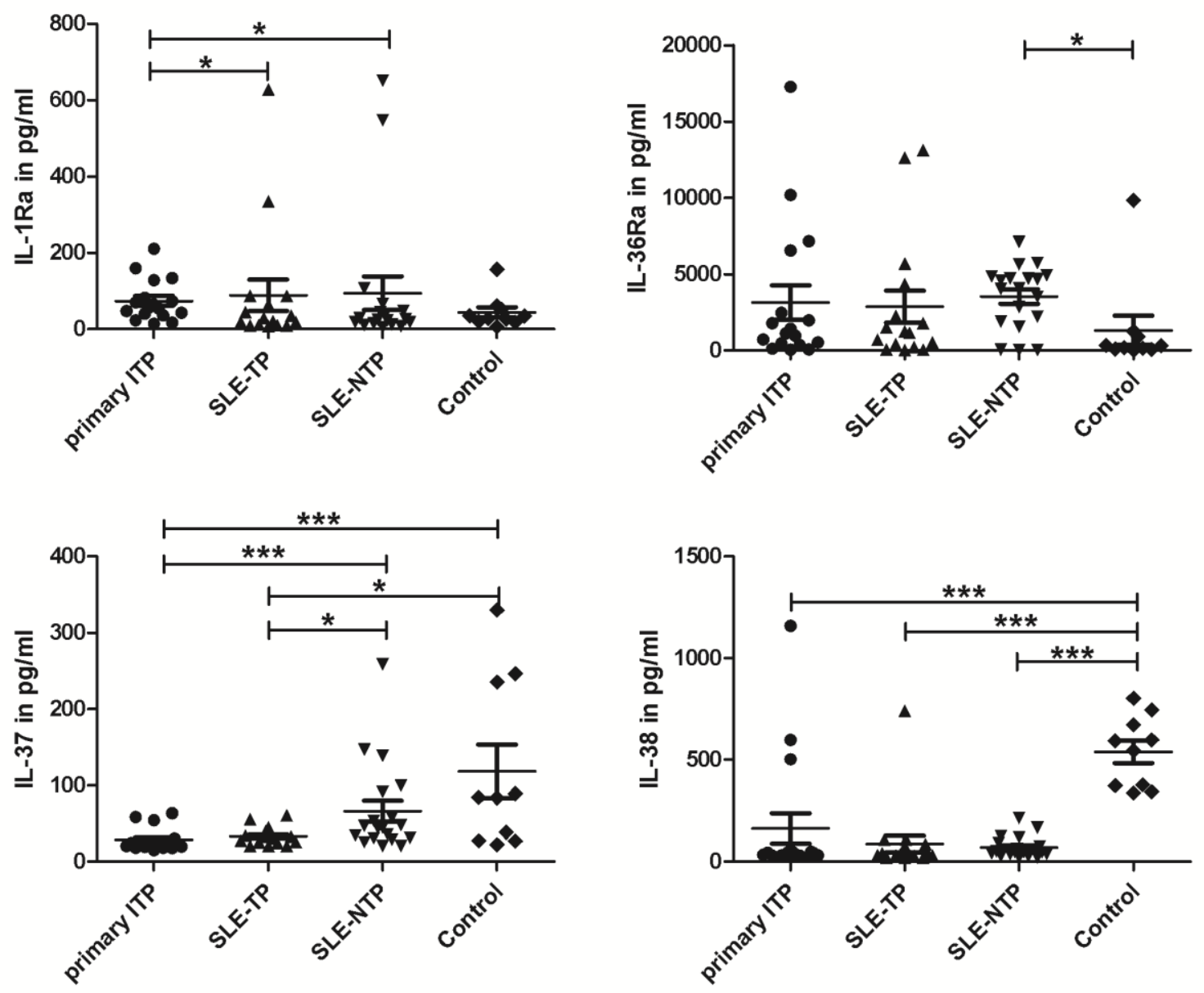
Figure 3 Serum IL-37 and IL-36 $\alpha$ correlated with platelet count.
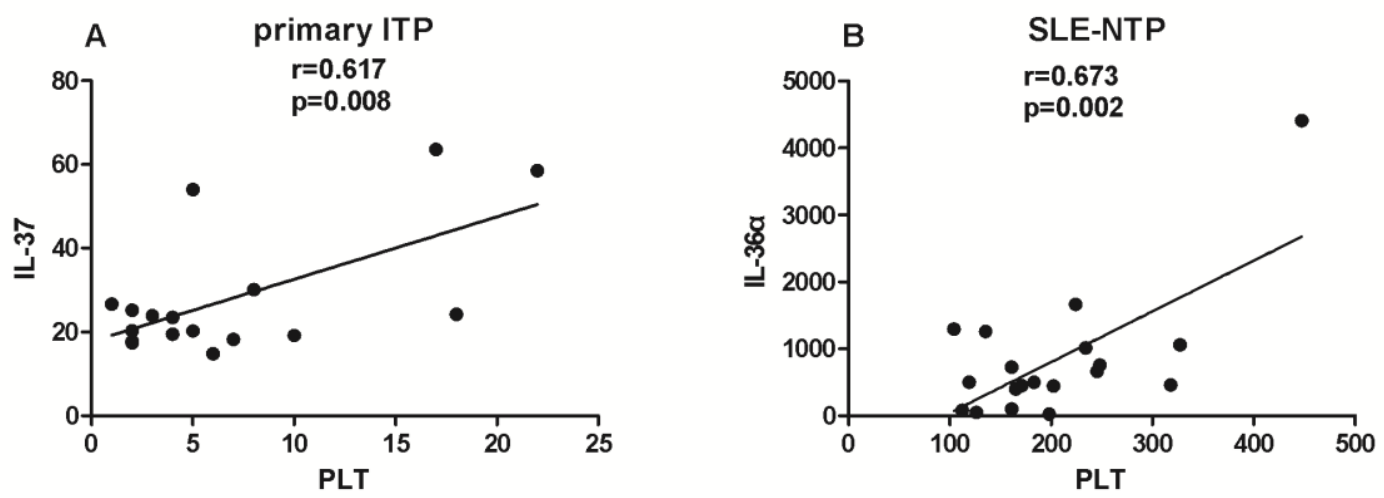
Figure 4 The mRNA expression of IL-1 cytokines in ITP patients, SLE-TP patients, SLE-NTP patients and healthy controls.
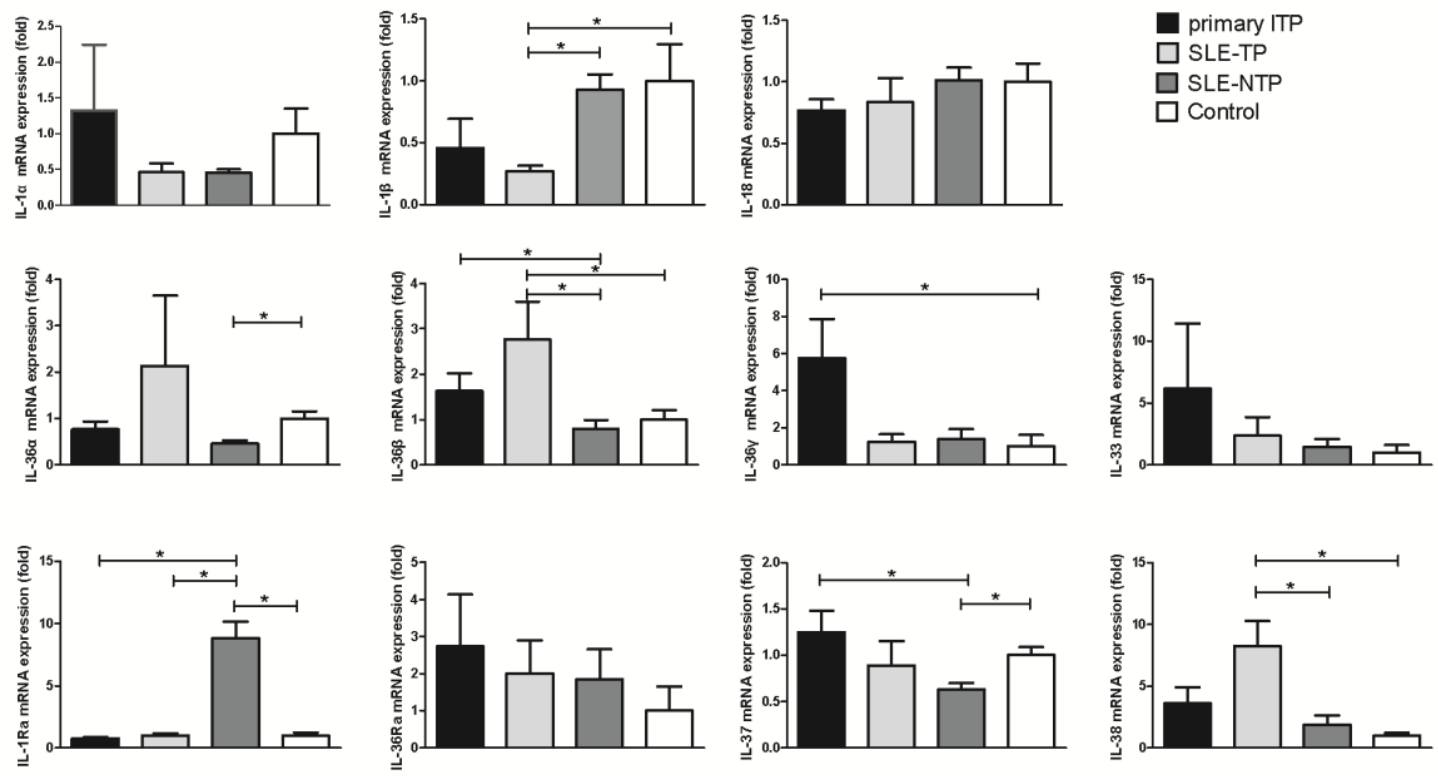


\section{Figure Legends}

Figure 1 The serum levels of IL-1 $\beta$, IL-18, IL-36 $\alpha$, IL-36 $\beta$, IL-36 $\gamma$ and IL-33 in ITP patients, SLE-TP patients, SLE-NTP patients and healthy controls. ITP patients have lower serum IL-1 $\beta$, IL-18, IL-36 $\alpha$, IL-36 $\beta$, IL-36 $\gamma$ and IL-33 as compared with SLE-TP, SLE-NTP patients and healthy controls. The levels of IL-1 $\beta$, IL-18, IL-36 $\alpha$,

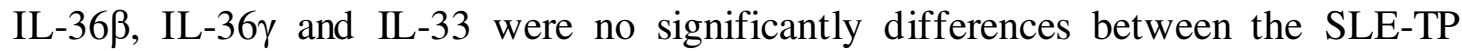
patients and SLE-NTP patients. The level of IL-33 was increased significantly in healthy controls than SLE-TP patients. ${ }^{*} p<0.05,{ }^{* *} p \leq 0.01$ and ${ }^{* * *} p \leq 0.001$ were labeled in the figure.

Figure 2 The levels of IL-1Ra, IL-36Ra, IL-37 and IL-38 in ITP patients, SLE-TP patients, SLE-NTP patients and healthy controls. ITP patients have lower IL-1Ra than SLE-TP and SLE-NTP patients. The level of IL-37 was lower in ITP patients and SLE-TP patients as compared with SLE-NTP patients and healthy controls. The level of IL-38 was lower in ITP patients, SLE-TP patients and SLE-NTP patients as compared with healthy controls. ${ }^{*} p<0.05,{ }^{* *} p \leq 0.01$ and ${ }^{* \star *} p \leq 0.001$ were labeled in the figure.

Figure 3 Serum IL-37 and IL-36 $\alpha$ correlated with platelet count. (A) Serum IL-37 positively correlated with platelet count in ITP patients. (B) Serum IL-36 $\alpha$ positively correlated with platelet count in SLE-NTP patients. The $p$ values less than 0.05 were labeled in the figure. Abbreviation: PLT, platelet count. 
Figure 4 The mRNA expression of IL-1 cytokines in ITP patients, SLE-TP patients, SLE-NTP patients and healthy controls. There were no significantly differences between ITP patients and SLE-TP patients. The expression of IL-1 $\beta$ mRNA was decreased significantly in SLE-TP patients as compared with SLE-NTP and healthy controls. The expression of IL-36 $\beta$ and IL-38 mRNA were increased significantly in SLE-TP patients as compared with SLE-NTP and healthy controls. The expression of IL-36 $\gamma$ mRNA was increased significantly in ITP patients than healthy controls. The expression of IL-Ra mRNA was increased significantly in SLE-NTP patients than ITP patients, SLE-TP patients and healthy controls. ${ }^{*} p<0.05$ were labeled in the figure. 

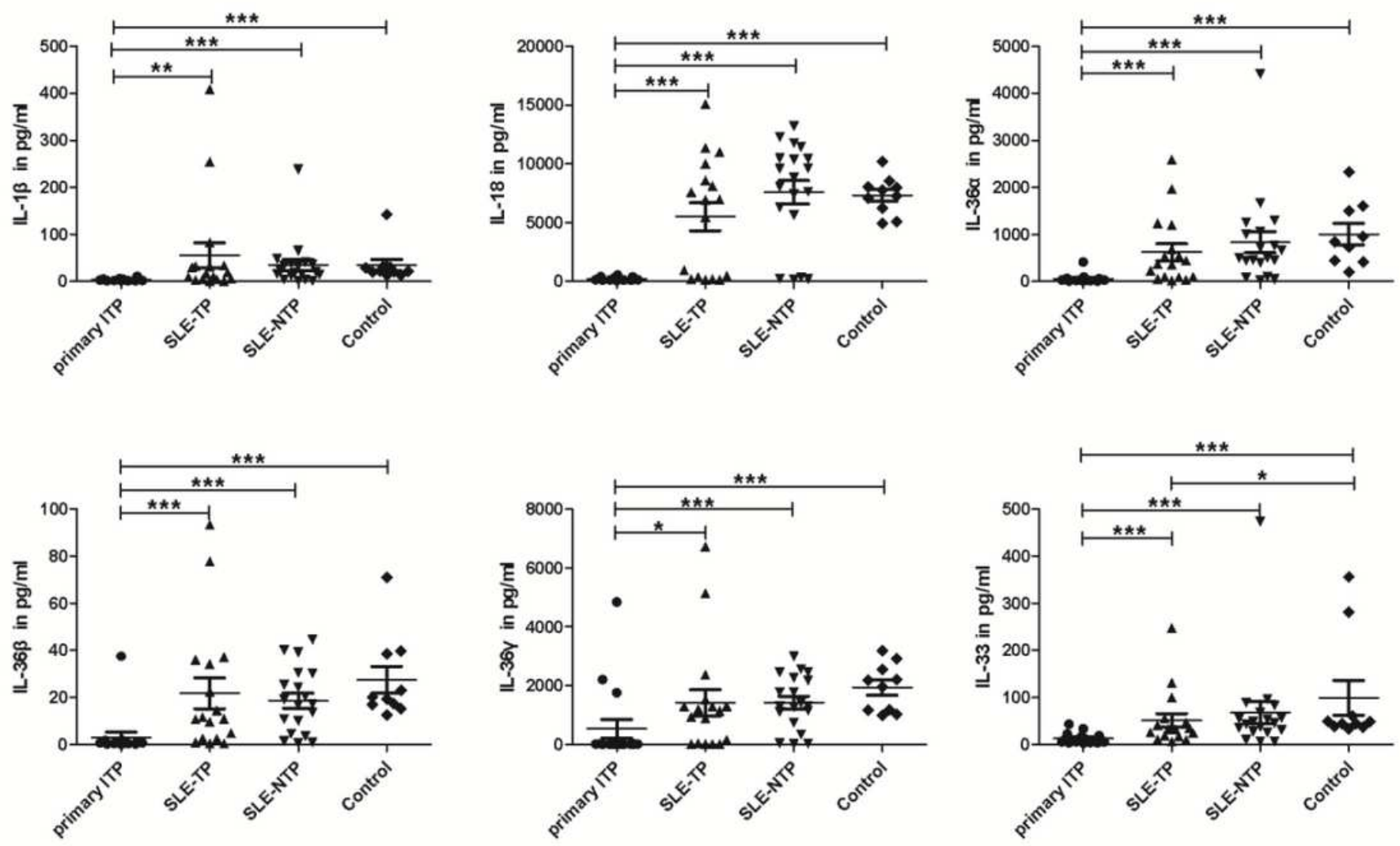

\section{Figure 1}

The serum levels of IL $1 \beta$ IL 18, IL $36 a, I L 36 \beta$, IL 36 y and IL 33 in ITP patients SLE TP patients, SLE NTP patients and healthy controls . ITP patients have lower serum IL $1 \beta$ IL 18, IL 36 a, IL $36 \beta$, IL 36 Y and IL 33 as compared with SLE TP, SLE NTP patients and healthy controls. T he levels of IL $1 \beta$ IL 18, IL $36 \mathrm{a}, \mathrm{IL} 36 \beta \mathrm{IL} 36$ y and IL 33 were no significant ly differences between the SLE TP patients and SLE N TP patients. T he level of IL 33 was increased significantl y in healthy controls than SLE TP pa tients . $p$ 0.05, p 0.01 and p 0.001 were labeled in the figure. 

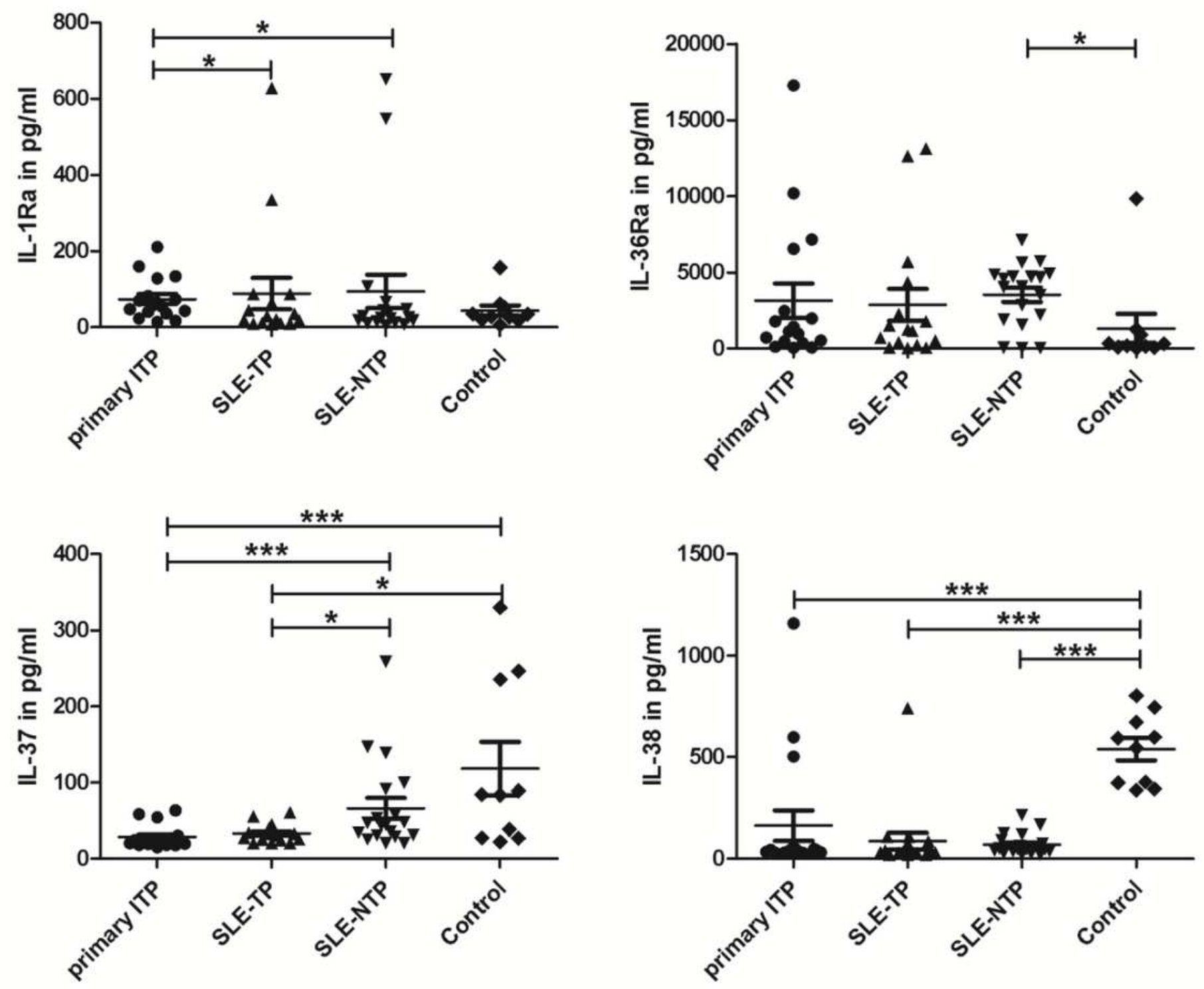

Figure 2

The levels of IL 1 Ra, IL 36Ra, IL 37 and IL 38 in ITP patients SLE TP patients, SLE NTP patients and healthy controls. ITP patients have lower IL 1Ra than SLE TP an d SLE NTP patients. T he level of IL 37 was lo wer in ITP patients and SLE TP patients as compared with SLE NTP patients and healthy controls T he level of IL 38 was lower in ITP patients SLE TP patients and SLE NTP patients as compared with healthy control s. p $0.05, \mathrm{p} 0.01$ and p 0.001 were labeled in the figure. 

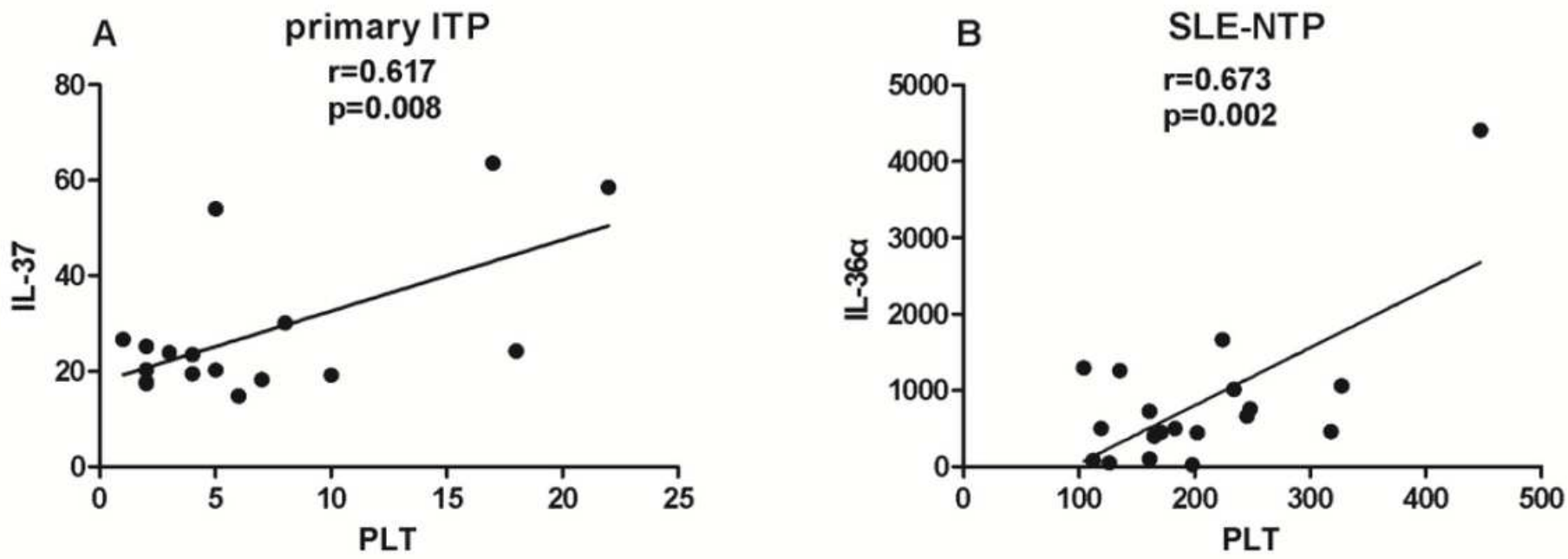

Figure 3

S erum IL 37 and IL 36 a correlated with platelet count. (A) Serum IL 37 positively correlated with platelet count in ITP patients. (B) Serum IL 36 a positively correlated with platelet count in S LE NTP patients. T he $p$ values less than 0.05 were labeled i $n$ the figure. Abbrevia tion PLT, $p$ latelet count.
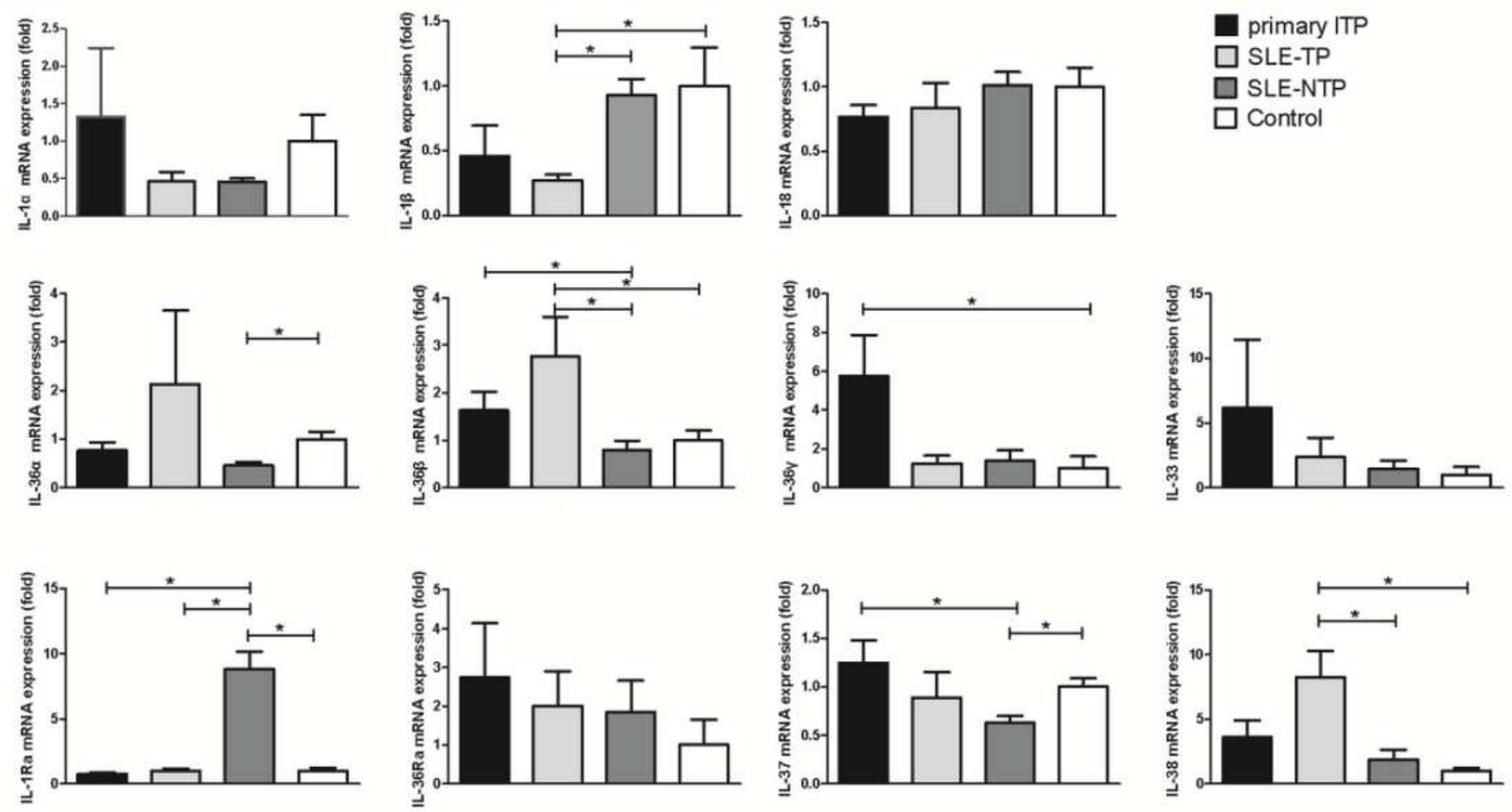

Figure 4

The mRNA expression of IL 1 cytokines in ITP patients SLE TP patients, SLE NTP patients and healthy controls. There were no significant ly diffe rences between ITP patients and SLE TP patients. The expression of IL $1 \beta$ mRNA was decreased significantly in SLE TP patients as compared with SLE NTP 
and healthy controls. The expression of IL $36 \beta$ and IL 38 mRNA were increased significantly in SLE TP patients as compared with SLE NTP and he althy controls. The expression of IL 36 y mRNA was increased significantly in ITP patients than healthy controls. The expression of IL Ra mRNA was increased significantly in SLE NTP patients than ITP patients, SLE TP patients and healthy controls. $p$ 0.05 were labeled in the figure. 